\title{
Duodenal Perforation as an Unusual Celiac Disease Presentation in Two Patients
}

\author{
Imad Absah ${ }^{\mathrm{a}, \mathrm{e}}$, Rayna M. Grothe ${ }^{\mathrm{a}}$, D. Dean Potter ${ }^{\mathrm{b}}$, Tsung-Teh Wuc ${ }^{\mathrm{c}}$, Joseph A. Murray ${ }^{\mathrm{d}}$
}

\begin{abstract}
Celiac disease (CD) is a common genetic autoimmune disease, triggered by gluten sensitivity. $\mathrm{CD}$ can present at any age in both children and adults after consuming gluten containing diet. Presentation of CD varies widely. There is paucity of data regarding emergency presentation of CD in the literature. Severe abdominal pain and gastric obstructions have been reported, but there are no reports of spontaneous duodenal perforation as of CD presentation. We searched the Mayo Clinic electronic medical records, between 1997 - 2011 for any cases of association between celiac disease and duodenal perforation. We identified two patients ( 7 yo M, 31 yo F). First patient presented with gastric obstruction due to duodenal perforation. Gastroduodenostomy was performed and elevated celiac serology few years after his initial presentation lead to the diagnosis of CD. Second patient had sudden acute RUQ abdominal pain and was found to have sign of visceral perforation on abdominal CT. Evaluation for anemia and rectal bleeding few years later showed positive CD markers that was confirmed by histology. Spontaneous duodenal perforation can be the presenting sign of CD. Celiac serology testing may be considered in patients presenting with spontaneous duodenal perforation.
\end{abstract}

Keywords: Celiac disease; Duodenal perforation; Surgical abdomen

\section{Introduction}

Celiac disease (CD) is a unique genetic autoimmune disease,

Manuscript accepted for publication November 13, 2012

${ }^{\mathrm{a} D e p a r t m e n t ~ o f ~ P e d i a t r i c ~ G a s t r o e n t e r o l o g y ~ a n d ~ H e p a t o l o g y, ~ M a y o ~}$ Clinic College of Medicine, USA

${ }^{b}$ Department of Pediatric Surgery, Mayo Clinic College of Medicine, USA

${ }^{\mathrm{c}}$ Department of Pathology, Mayo Clinic College of Medicine, USA

${ }^{\mathrm{d}}$ Department of Medicine, Division of Gastroenterology and

Hepatology, Mayo Clinic College of Medicine, USA

${ }^{\mathrm{e}}$ Corresponding author: Imad Absah, 200 First Street SW, Rochester,

Mn 55905, USA.Email: absah.imad@mayo.edu

doi: http://dx.doi.org/10.4021/jmc1006e because its environmental triggering agent gluten is known. Gluten, the protein found in wheat, barley and rye, induces immune response causing flattening of the intestinal villi and eventually compromises the ability of the patient to absorb nutrients through the intestine.

$\mathrm{CD}$ is a common autoimmune disease seen in both adults and children; it affects $1 \%$ of the population [1]. Clinical manifestations of CD differ by age; hence, malabsorption has been regarded as the classic manifestation. This can manifest as growth failure, weight loss, deficiency states, as well as diarrhea or steatorrhea. Extra intestinal manifestations of $\mathrm{CD}$, such as skin rash, neurologic symptoms and chronic anemia, have been reported. Less-commonly reported manifestations are direct consequences of inflammation, including ulceration, intussusception, and papillary stenosis. While first described over 70 years ago, it is only recently that these inflammatory presentations of $\mathrm{CD}$ have received more attention. Indeed, it is estimated that $3 \%$ of patients presenting with acute abdominal pain may have underlying celiac disease [2]. Another related observation was the association between the papillary stenosis that resulted in recurrent acute pancreatitis in undiagnosed celiac patients [3]. CD also made up a small but significant portion of those patients who presented with intussusception [4]. These examples illustrate that the consequences of the inflammatory nature of celiac disease can lead to surgical abdominal pain.

Spontaneous duodenal perforation has not been to our knowledge previously reported in celiac disease. Herein we report two cases of celiac disease presenting with duodenal

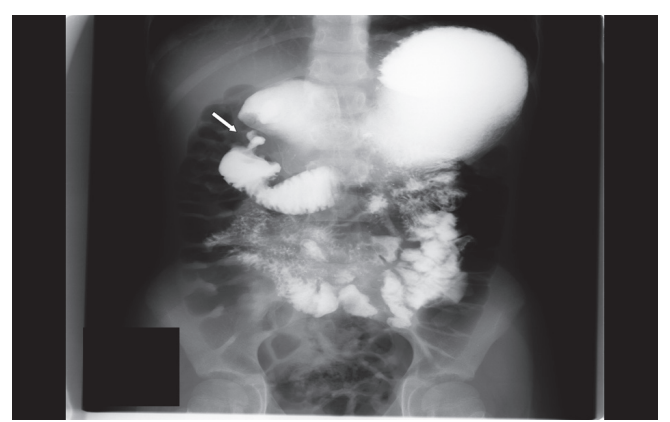

Figure 1. Upper GI barium study showing duodenal bulb dilatation and possible duodenal obstruction. 


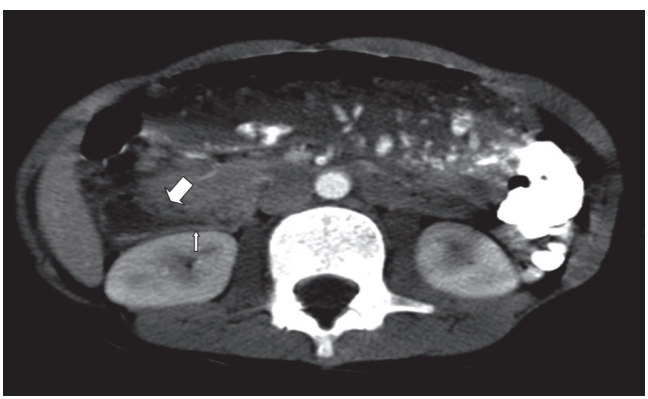

Figure 2. CT image showing narrowed descending duodenal lumen with tissue inflammation and thickness around the mid-duodenum suspicious of annular pancreas or intestinal perforation.

perforation at 7 and 31 years of age, respectively. Both patients were subsequently found to have celiac disease.

\section{Case Report}

We searched the Mayo Clinic electronic medical records between 1997 - 2011 for all cases of CD associated with duodenal perforation. We identified two cases ( 7 yo M, 31 yo F). The original surgical pathology specimens were re-reviewed by a GI pathologist (TW) to look for presence of CD or any other reason for their duodenal perforation. This study was approved by the Mayo Clinic Institutional Review Board.

\section{Case 1}

A 7-year-old male presented with 8 weeks history of early satiety, abdominal pain, and nonbilious vomiting. On initial assessment he was anemic with hemoglobin $(7.8 \mathrm{~g} /$ $\mathrm{dL})$ and low ferritin $(5 \mu \mathrm{g} / \mathrm{L})$. His total serum IgA was not done and his celiac screening showed negative endomysial $\mathrm{Ab}$, and tTG Ig A value of 5 (negative < 4). His upper GI barium study showed duodenal bulb dilatation and possible

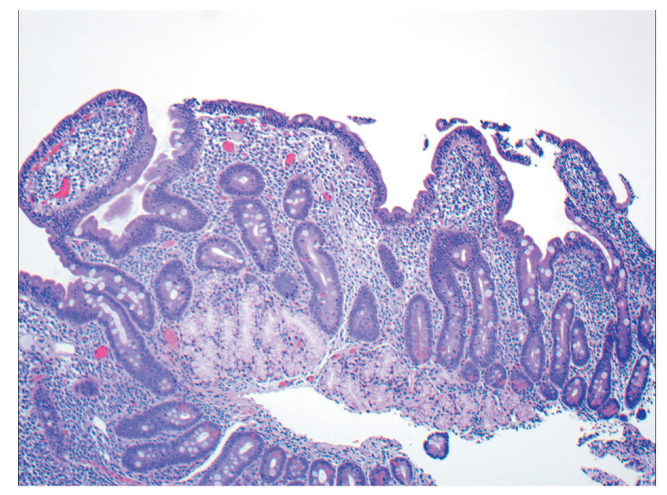

Figure 3. Complete villous atrophy, crypt hyperplasia and intraepithelial lymphocytosis.

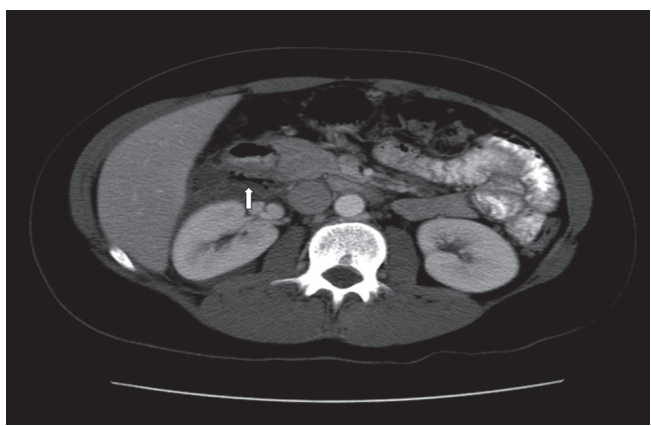

Figure 4. CT image showing free gas and fluid collection in the abdomen posterior to the duodenal bulb with inflammatory changes throughout the mesenteric fat suggestive of intestinal perforation.

duodenal obstruction (Fig. 1), and then abdominal computed tomography (CT) showed narrowed descending duodenal lumen with tissue inflammation and thickness around the mid-duodenum suspicious of annular pancreas (Fig. 2). The upper endoscopy (EGD) showed normal cardia, corpus, and fundus. However, multiple aphthous ulcerations and superficial erosions were noted in the antrum. Duodenal bulb was dilated with normal mucosa; the scope couldn't be advanced to the descending duodenum due to luminal obstruction.

Gastric biopsies showed mild active chronic gastritis, with occasional Helicobacter pylori (H. Pylori). Duodenal biopsies showed mild chronic duodenitis with partial villous atrophy. This partial villous atrophy was attributed to chronic peptic duodenitis. Due to inability of passing the endoscope beyond the duodenal bulb and the unclear etiology of his duodenal obstruction laparoscopic abdominal exam was performed. Laparoscopy discovered dense chronic inflammatory tissue across the duodenal bulb suggestive of a previous perforation. Patient underwent gastroduodenostomy and then discharged home on H. Pylori treatment. He remained asymptomatic until age 13 years. He was seen after a blunt trauma to the abdomen during skiing. He was hospitalized for severe abdominal pain and leukocytosis (WBC

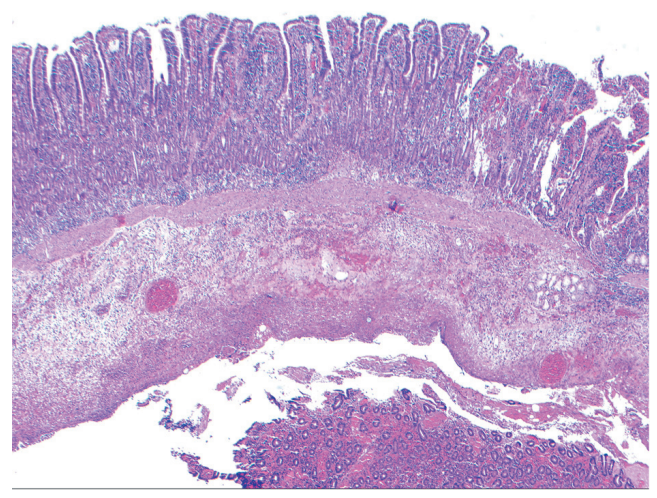

Figure 5. Resected duodenal diverticulum with acute duodenitis with features suggesting celiac disease. 
20.3). The rest of his blood works, including ALT, amylase, lipase, and gastrin level were normal. CD serologic markers were not done. At this time, abdominal CT showed free fluid and soft tissue thickening around the antrum, greater curvature of the stomach, and the duodenum, but no evidence of free air and bowel perforation. Upper GI barium study excluded any leak, so EGD was performed. He had multiple superficial antral erosions with normal duodenal mucosa. Histology showed H. Pylori negative moderate chronic gastritis and partial duodenal villous atrophy (villous crypt ratio $2: 1$ ) without significant intraepithelial lymphocytosis. These findings were attributed again to chronic peptic disease. $\mathrm{He}$ was treated with proton pump inhibitor (PPI) and remained asymptomatic for about a year. He stopped his PPI therapy and slowly started complaining of mild abdominal pain. Follow-up blood work showed normal hemoglobin (14.8 g/ $\mathrm{dL})$, low ferritin $(19 \mu \mathrm{g} / \mathrm{L})$, and normal serum IgA (63 mg/ dL). His CD serologic markers came back positive, Anti tTG $\operatorname{IgA}$ and $\operatorname{IgG}$ levels were 11.9 and 100 , respectively. This was followed with EGD that showed malabsorptive pattern consistent with CD (Fig. 3). Based on blood work and biopsies, he was diagnosed with $\mathrm{CD}$ and started on gluten-free diet with vitamin and iron supplements. Repeat titers at 3 months showed Anti tTG IgA and IgG levels of 2.2 and 41.3, respectively. A follow up phone call documented that his abdominal pain is gone since he started his gluten free diet.

\section{Case 2}

A previously healthy 31-year-old female presented with 4-day history of abdominal pain, nausea, vomiting, and fever. Her initial assessment showed anemia with hemoglobin $(10.5 \mathrm{G} / \mathrm{dL})$, mild leukocytosis, and normal liver and pancreatic enzymes. CD serologic markers were not performed. Abdominal ultrasound (US) and HIDA (Hepatobiliary Iminodiacetic Acid) scan were normal. Patient's abdominal pain and fever persisted; she was given intravenous antibiotics and then transferred to Mayo clinic for further work up. Upon admission she had persistent abdominal discomfort; her CT showed free gas and fluid collection in the abdomen posterior to the duodenal bulb with inflammatory changes throughout the mesenteric fat (Fig. 4). The CT findings suggested visceral perforation with differential diagnosis, including retrocecal appendicitis with perforation versus a perforated duodenal ulcer. The patient underwent abdominal exploration and excision of perforated duodenal diverticulum, cholecystectomy, appendectomy and drainage of retroperitoneal abscess. Pathology report of the resected duodenum showed mural severe acute inflammation consistent with diverticular rupture. No further work up was done and patient was treated and discharged home. Five years later she was reevaluated because of rectal bleeding. During that visit her history was significant for refractory chronic iron deficiency anemia and history of miscarriage due to severe fetal neural tube defect. Her screening blood work showed low hemoglobin $(8.8 \mathrm{~g} / \mathrm{dL})$ and positive $\mathrm{CD}$ serologic markers. Anti tTG IgA and IgG levels were $>150$ and 4 respectively. She had normal EGD and colonoscopy exam, but her duodenal histology showed increased intraepithelial lymphocytes (40 lymphocytes per 100 epithelial cells), total villous atrophy, crypt hyperplasia, and chronic inflammation. Retrospective review of the resected duodenal specimen at the initial presentation also showed histologic features of CD (Fig. 5). Patient was then diagnosed with $\mathrm{CD}$ and started on gluten-free diet. Patient was seen recently for a follow-up visit, she has been doing very well and her follow-up work up included upper endoscopy and celiac serology titers. Her anemia resolved with Hemoglobin (14.3 g/dL), her CD markers were normal with anti tTG IgA (2.3), and her histology assessment showed resolution of her villous atrophy and presence of intraepithelial lymphocytes.

\section{Discussion}

Prior to the wide use of proton pump inhibitors in 1980s perforation of duodenal ulcer was the most common cause of small bowel (SB) perforation. Currently ERCP (endoscopic retrograde cholangiopancreatography) is the most common cause of SB perforation. Other causes of perforation include infections (tuberculosis and cytomegalovirus), Crohn's disease, ischemia, injury from radiation therapy, cancer (lymphoma, adenocarcinoma, etc.), and swallowed foreign bodies [5]. Celiac disease has not been reported as a cause of spontaneous duodenal perforation, so it is not on the differential diagnosis list for cases presenting with idiopathic duodenal perforation.

We report two cases of duodenal perforation that were followed subsequently by a diagnosis of $\mathrm{CD}$. These illustrate a likely severe consequence of the inflammatory nature of $\mathrm{CD}$ that could occur in the absence of classic malabsorptive findings. This may be another manifestation that is due to inflammation that can include ulceration, strictures, obstruction, and papillary stenosis causing recurrent acute pancreatitis, and possibly even bleeding [3, 6, 7].

Celiac disease may be overlooked in the context of emergency surgery carried out due to intestinal obstruction or, in our case, perforation. This has previously been reported in circumstances of obstruction caused by malignancies of the small intestine. Surgeons and surgical pathologists need to be aware of and familiar with diseases, including celiac disease that could have led to the acute event which precipitated surgery and possibly surgical sampling. The difficulties of interpretation are compounded often by the acute changes associated with perforation or obstruction. However, the presence of intraepithelial lymphocytosis would not be a typical acute effect.

Small bowel ulceration can result from variety of eti- 
ologies, such as IBD, acid-induced, chemical-induced (NSAIDS), congenital malformation and neoplastic diseases. It is unclear if the presentation of duodenal perforation in these two cases was due to H. pylori in the first patient and congenital duodenal diverticula in the second patient or if it was an unusual presentation of CD.

H. pylori is still a controversial topic in children. Recent data suggested that in childhood, H. pylori is associated with predominant antral gastritis and duodenal ulcers [8]. This is very similar to the initial endoscopy findings in our patients. Perforation is a known complication of duodenal ulcers and, in many cases, it presents without previous dyspepsia [9]. Successful eradication of H. pylori markedly reduces the rate of recurrence of duodenal ulcers in affected children. H. pylori was treated in our patient, but he continued to have stomach and duodenal ulceration despite being H. pylori negative by histology and stool antigen. Having negative celiac serology (endomysial Ab and tTG) doesn't exclude CD knowing that this is not always positive, especially in patients with partial villous atrophy [10]. This makes it more likely that both his duodenal ulceration and perforation were due to his CD. Ulceration of small bowel is not yet well understood; some authors think it's related to the long-term destruction of the mucosa by the gluten exposure or due to bacterial invasion of the damaged mucosa, but other data suggested that ulcer can occur even in patients on a gluten-free diet [11]. On the other hand, in adults with celiac disease, the presence of small bowel ulceration always increases the suspicion of $\mathrm{T}$ cell related lymphoma [12]. It has been reported that adult patients with CD and small bowel ulceration have a poor response to gluten-free diet [13]. This seems to be different in pediatric patients; our patient responded very well to the gluten exclusion, which is consistent with a report by Eltumi et al, who described two cases of pediatric patients with abdominal pain and diarrhea who where found to have extensive intestinal ulceration and responded to gluten-free diet. There is also a recent case of a 15-month-old child with failure to thrive who was found to have duodenal ulceration due to $\mathrm{CD}$ which responded to gluten exclusion [14].

In the second patient, exploratory laparotomy found perforated duodenal diverticulum. Duodenal diverticulum is still not a well-understood pathology' they are relatively common and estimated to be $5-22 \%$ in healthy individuals. Diverticula are usually located along the pancreatic or mesenteric border, mainly in the second part of the duodenum near the ampulla of Vater [15]. Duodenal diverticula are usually asymptomatic. There is no pathognomonic presentation, but different manifestations have been reported. The most common is inflammation, GI bleeding, pancreatitis, and bile duct obstruction. Duodenal perforation is a rare presentation that is associated with a mortality rate up to $20 \%$ [16]. None of the reports have linked CD to the development of duodenal diverticula; however, there was one report by Saml- off et al about a CD resulting in multiple jejunal diverticula [17]. It's unclear if the finding of duodenal diverticula in our patient was incidental or if the duodenal inflammation and ulceration due to $\mathrm{CD}$ caused intraluminal diverticulum that was complicated by perforation. When our GI pathologist reviewed the histology of the resected diverticulum, he found histologic findings' suggestive of CD that likely preceded her duodenal perforation.

Finally, both patients had spontaneous duodenal perforation requiring surgical intervention. The diagnosis of $\mathrm{CD}$ was delayed and confirmed later by histologic and serologic testing. By combining these two cases with the few case reports in the literature, we now know that CD can present as acute surgical abdomen like duodenal perforation. For that in cases of duodenal perforation that is not associated with acid hypersecretion or NSAIDs use think about Celiac disease as a cause. It will be reasonable to consider checking celiac serology in patients presenting spontaneous duodenal perforation. This might help identify CD earlier and prevent further inflammatory complications.

\section{Conclusion}

Whilst undoubtedly rare, duodenal perforation in these two patients with undiagnosed celiac disease affirms the need to be aware of the acute and indeed serious manifestations of celiac disease.

\section{Abbreviations}

CD: celiac disease; EGD: esophgogastroduodenal endoscopy; PPI: proton pump inhibitor; UGI: barium study of the upper gastrointestinal system; CT: Computed tomography; US: ultra sound; HIDA: Hepatobiliary Iminodiacetic Acid.

\section{References}

1. Maki M, Mustalahti K, Kokkonen J, Kulmala P, Haapalahti M, Karttunen T, Ilonen J, et al. Prevalence of Celiac disease among children in Finland. N Engl J Med. 2003;348(25):2517-2524.

2. Sanders DS, Hopper AD, Azmy IA, Rahman N, Hurlstone DP, Leeds JS, George RR, et al. Association of adult celiac disease with surgical abdominal pain: a case-control study in patients referred to secondary care. Ann Surg. 2005;242(2):201-207.

3. Patel RS, Johlin FC, Jr., Murray JA. Celiac disease and recurrent pancreatitis. Gastrointest Endosc. 1999;50(6):823-827.

4. Sanders DS, Azmy IA, Kong SC, Lee FK. Symptomatic small bowel intussusception: a surgical opportunity to diagnose adult celiac disease? Gastrointest Endosc. 2004;59(1):161-162. 
5. Tavakkoli A, Ashley SW, Zinner MJ. Small intestine. Schwartz's Principles of Surgery. McGraw-Hill, 2005.

6. Schweiger GD, Murray JA. Postbulbar duodenal ulceration and stenosis associated with celiac disease. Abdom Imaging. 1998;23(4):347-349.

7. Fine KD. The prevalence of occult gastrointestinal bleeding in celiac sprue. N Engl J Med. 1996;334(18):11631167.

8. Blecker U. Helicobacter pylori-associated gastroduodenal disease in childhood. South Med J. 1997;90(6):570576; quiz 577.

9. Greenfield LJ, Proctor MC. Attitudes toward retirement. A survey of the American Surgical Association. Ann Surg. 1994;220(3):382-389; discussion 387-390.

10. Katz KD, Rashtak S, Lahr BD, Melton LJ, 3rd, Krause PK, Maggi K, Talley NJ, et al. Screening for celiac disease in a North American population: sequential serology and gastrointestinal symptoms. Am J Gastroenterol. 2011;106(7):1333-1339.
11. Venturatos SG, Hines C, Jr., Blalock JB. Ulceration of the small intestine in a patient with celiac disease. South Med J. 1984;77(4):520-522.

12. Mills PR, Brown IL, Watkinson G. Idiopathic chronic ulcerative enteritis. Report of five cases and review of the literature. Q J Med. 1980;49(194):133-149.

13. Robertson DA, Dixon MF, Scott BB, Simpson FG, Losowsky MS. Small intestinal ulceration: diagnostic difficulties in relation to coeliac disease. Gut. 1983;24(6):565-574.

14. Mones RL, Mercer GO. Ulcerative duodenitis in a child with celiac disease. J Pediatr. 2011;158(5):857.

15. Cattell RB, Mudge TJ. The surgical significance of duodenal diverticula. N Engl J Med. 1952;246(9):317-324.

16. Psathakis D, Utschakowski A, Muller G, Broll R, Bruch HP. Clinical significance of duodenal diverticula. J Am Coll Surg. 1994;178(3):257-260.

17. Samloff IM, Schenk EA. Celiac disease and multiple jejunal diverticulosis. Am J Dig Dis. 1967;12(2):189-197. 